\title{
LATTICES OF SUBALGEBRAS OF LEIBNIZ ALGEBRAS
}

\author{
DONALD W. BARNES
}

\begin{abstract}
I describe the lattice of subalgebras of a one-generator Leibniz algebra. Using this, I show that, apart from one special case, a lattice isomorphism $\phi: \mathcal{L}(L) \rightarrow \mathcal{L}\left(L^{\prime}\right)$ between Leibniz algebras $L, L^{\prime}$ maps the Leibniz kernel Leib $(L)$ of $L$ to $\operatorname{Leib}\left(L^{\prime}\right)$.
\end{abstract}

\section{INTRODUCTION}

A lot is known about the lattices of subalgebras of Lie algebras. See for example, Barnes [1], [2], Goto [4], Towers [5], [6], [7]. In this paper, I look at some basic results needed to extend these results to Leibniz algebras.

In the following, $L, L^{\prime}$ are finite-dimensional (left) Leibniz algebras over the field $F$. I denote by $\langle a, b, \ldots\rangle$ the subspace spanned by the listed elements and by $\operatorname{alg}\langle a, b, \ldots\rangle$ the subalgebra they generate. The Leibniz kernel of $L$ is the subspace $\operatorname{Leib}(L)=\left\langle x^{2} \mid x \in L\right\rangle$ spanned by the squares of the elements of $L$. By Barnes [3, Lemma 1.1], it is a 2-sided ideal and $L / \operatorname{Leib}(L)$ is a Lie algebra.

Since the main aim of this paper is to show that (apart from one exceptional case, $\operatorname{Leib}(L)$ can be recognised from lattice properties alone, we say of a subalgebra $U$ of $L$, that $U$ is recognisable if, from properties of the lattice $\mathcal{L}(L)$, it can be shown that $U \subseteq \operatorname{Leib}(L)$, that is, if for every lattice isomorphism $\phi: \mathcal{L}(L) \rightarrow \mathcal{L}\left(L^{\prime}\right)$, we have $\phi(U) \subseteq \operatorname{Leib}\left(L^{\prime}\right)$.

There is one case in which $\operatorname{Leib}(L)$ is not recognisable.

Example 1.1. Let $L=\operatorname{alg}\langle a\rangle=\left\langle a, a^{2}\right\rangle$ with $a^{3}=a^{2}$. Put $b=a-a^{2}$ and $v=a^{2}$. Then $b^{2}=v^{2}=v b=0$ and $b v=v$. Then $\operatorname{Leib}(L)=\langle v\rangle$ and $\mathcal{L}(L)$ is

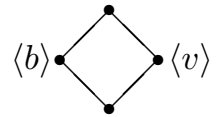

The map $\phi: \mathcal{L}(L) \rightarrow \mathcal{L}(L)$ which interchanges $\langle b\rangle$ and $\langle v\rangle$ is clearly a lattice automorphism.

The lattice of Example 1.1 will be called the diamond lattice and an algebra with this as its subalgebra lattice will be called a diamond algebra. The lattice of all subspaces of a vector space over $F$ will be called a vector space lattice.

Lemma 1.2. Let $D$ be a diamond algebra. Then there exist $b, v \in D$ such that $D=\langle b, v\rangle$ and $b^{2}=v^{2}=v b=0$ and $b v=v$.

Proof. A Leibniz algebra $L$ which is not a Lie algebra has a proper subalgebra Leib $(L)$, so an algebra without proper subalgebras is a 1-dimensional Lie algebra. $D$ has two proper subalgebras, $B$ and $V$ say. As not every 1-dimensional subspace

2010 Mathematics Subject Classification. Primary 17A32.

Key words and phrases. Leibniz algebras, lattices. 
of $D$ is a subalgebra, $D$ is not a Lie algebra, so one of these, $V$ say is $\operatorname{Leib}(L)$. Take generators $b, v$ of $B, V$ respectively. Then $b^{2}=v^{2}=v b=0$ and $b v=\lambda v$ for some $\lambda \in F, \lambda \neq 0$. We replace $b$ with $b / \lambda$.

To avoid repeated descriptions of notations, I use the following convention. Whenever we have a subalgebra $U$ of $L, U^{\prime}$ denotes the subalgebra $\phi(U)$ of $L^{\prime}$ under the lattice isomorphism $\phi: L \rightarrow L^{\prime}$. For an element $a \in L, a^{\prime}$ denotes a generator of $\operatorname{alg}\langle a\rangle^{\prime}$. (We shall see later, Lemma 2.7, that $\operatorname{alg}\langle a\rangle^{\prime}$ is a one-generator algebra. This holds trivially if $\operatorname{dim} \operatorname{alg}\langle a\rangle=1$.) If $A \supseteq B$ are subalgebras of $L, \mathrm{I}$ denote by $A \div B$ the lattice interval consisting of all subalgebras $C$ with $A \supseteq C \supseteq B$.

\section{ONE-GENERATOR ALGEBRAS}

Consider the one-generator Leibniz algebra $L=\left\langle a, a^{2}, \ldots, a^{n}\right\rangle$. We have here a vector space $V=\left\langle a^{2}, \ldots, a^{n}\right\rangle$ acted on by a linear transformation $\theta: V \rightarrow V$ such that $V$ is generated as $F[\theta]$-module by the element $a^{2}$. Let $f(x)=x^{r} g(x)$ be the characteristic polynomial of $\theta$. Then $f(x)$ is also the minimum polynomial of $\theta$. Put $V_{1}=\theta^{r} V$. Then $g(x)$ is the minimum polynomial of $\theta \mid V_{1}$.

Put $h(x)=(g(x)-g(0)) / x g(0)$ and $b=a+h(\theta) a^{2}$. Then

$$
b^{r+2}=a^{r+2}+\theta^{r+1} h(\theta) a^{2}=a^{r+2}+(g(\theta)-g(0)) a^{r+2} / g(0)=0 .
$$

Let $B$ be the subalgebra generated by $b$. Then $B^{2}$ is the only maximal subalgebra of $B$ and $\mathcal{L}(B)$ consists of $B$ and all the subspaces of $B^{2}$. If $a$ is not nilpotent, then $B$ is a proper subalgebra of $L$ not contained in the maximal subalgebra $V$, so $V$ is not the only maximal subalgebra of $L$.

Lemma 2.1. Let $B=\operatorname{alg}\langle b\rangle$ be a subalgebra generated by a nilpotent element $b$. Then $B \simeq B^{\prime}$ and $B^{2}=\operatorname{Leib}(B)$ is recognisable.

Proof. $B^{2}$ is the only maximal subalgebra of $B$, so $W=\phi\left(B^{2}\right)$ is the only maximal subalgebra of $B^{\prime}$. There exists $c \in B^{\prime}, c \notin W$. Since $c$ is not contained in any maximal subalgebra of $B^{\prime}$, we have $\operatorname{alg}\langle c\rangle=B^{\prime}$. Since $W$ is the only maximal subalgebra of $B^{\prime}, c$ is nilpotent. Since the maximal chains of $\mathcal{L}(B)$ and $\mathcal{L}\left(B^{\prime}\right)$ have the same length, we have $B \simeq B^{\prime} . \operatorname{Leib}(B)=B^{2}$ and $\phi(\operatorname{Leib}(B))=W=$ $\operatorname{Leib}\left(B^{\prime}\right)$.

Lemma 2.2. Suppose $c \notin V$ is nilpotent, then $\operatorname{alg}\langle c\rangle=B$.

Proof. Since $c \notin V$, we have $c=\lambda b+b_{1}+v$ for some $\lambda \in F, \lambda \neq 0, b_{1} \in B^{2}$ and $v \in V_{1}$. Since $\theta$ is non-singular on $V_{1}, v=0$ and $c \in B$. But $c$ is not in the only maximal subalgebra of $B$, so $\operatorname{alg}\langle c\rangle=B$.

To determine the invariant subspaces, we use the prime power factorisation $g(x)=p_{1}^{r_{1}}(x) \ldots p_{k}^{r_{k}}(x)$ where the $p_{i}(x)$ are distinct irreducible polynomials. Since $V$ and so also $V_{1}$ is generated as $F[\theta]$-module by a single element, the only invariant subspaces of $V_{1}$ are the spaces

$$
V_{s_{1}, \ldots, s_{k}}=\left\{v \in V \mid p_{1}^{s_{1}}(\theta) \ldots p_{k}^{s_{k}}(\theta) v=0\right\}=\theta^{r} p_{1}^{r_{1}-s_{1}}(\theta) \ldots p_{k}^{r_{k}-s_{k}}(\theta) V,
$$

for $s_{i} \leq r_{i}$. Put $U_{s_{1}, \ldots, s_{k}}=B+V_{s_{1}, \ldots, s_{k}}$.

Theorem 2.3. Let $L=\operatorname{alg}\langle a\rangle$. Then the $U_{s_{1}, \ldots, s_{k}}$ are the only subalgebras of $A$ not contained in $V$. The lattice interval $L \div B$ is the lattice product of $k$ chains of lengths $r_{1}, \ldots, r_{k}$. 
Proof. Let $C$ be a subalgebra of $L$ not contained in $V$. Then $C$ has an element $c=b+b_{1}+v$ where $b_{1} \in B^{2}$ and $v \in V_{1}$. As above, we obtain a nilpotent element $c_{0}=c+w \in C$ where $w \in V$. By Lemma $2.2, c_{0}$ generates $B$. Thus $B \subseteq C$. It follows also that $v \in C$, and the invariant subspace generated by $v$ is contained in $C$. It follows that $C=U_{s_{1}, \ldots, s_{k}}$ for some $s_{1}, \ldots, s_{k}$. That the lattice interval is the product of chains as described follows.

To illustrate this, I consider the case $r=0, k=1, r_{1}=1$ and $p_{1}(x)=x-1$.

Example 2.4. Let $L=\left\langle b, v_{1}, v_{2}\right\rangle$ with $b^{2}=v_{i} x=0$ for all $x \in L$ and $b v_{1}=v_{1}+v_{2}$, $b v_{2}=v_{2}$. Then $L=\operatorname{alg}\left\langle b+v_{1}\right\rangle$ and $\mathcal{L}(L)$ is

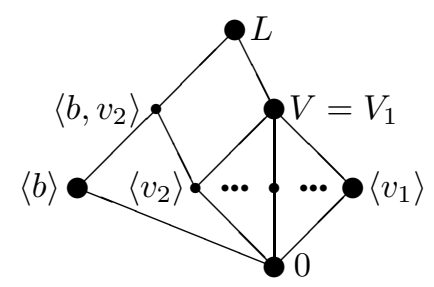

Observe that the emphasised points $L, V_{1},\langle b\rangle,\left\langle v_{1}\right\rangle, 0$ form a sublattice, and that $\mathcal{L}(L)$ is not modular. Indeed, for any one-generator algebra with $\operatorname{dim}\left(V_{1}\right)>1$, taking $v_{1}$ an element which generates $V_{1}$ under the action of $\theta$, we obtain in this way the standard non-modular lattice.

Definition 2.5. The signature of the one-generator Leibniz algebra $L$ is the list $\left[r\left|r_{1}, \ldots, r_{k}\right| d_{1}, \ldots, d_{k}\right]$ where $d_{i}$ is the degree of the irreducible polynomial $p_{i}(x)$. If $k=1$, we call $L$ a single-chain algebra.

Clearly, from the signature and knowledge of the field $F$, one can reconstruct $\mathcal{L}(L)$. The algebra $L$ of Example 2.4 is a single-chain algebra with signature $[0|2| 1]$.

Lemma 2.6. Suppose that $L$ is a single-chain algebra. Then $L^{\prime}$ is a single-chain algebra with the same signature as $L$. If $\operatorname{dim}(L)>2$, then $\phi(\operatorname{Leib}(L))=\operatorname{Leib}\left(L^{\prime}\right)$.

Proof. $L$ has exactly two maximal subalgebras, so $L^{\prime}$ has exactly two maximal subalgebras. A vector space cannot be the set union of two proper subspaces, so there exists $a^{\prime} \in L^{\prime}$ which is not contained in any maximal subalgebra. Thus $\operatorname{alg}\left\langle a^{\prime}\right\rangle=L^{\prime}$. Let $\left[r\left|r_{1}\right| d_{1}\right]$ be the signature of $L$. Let $M$ be maximal subalgebra containing $B$.

The lattice of $V$ is the vector space lattice of dimension $\operatorname{dim}(V)=r+r_{1} d_{1}$ and it follows that $\operatorname{dim}\left(L^{\prime}\right) \geq 1+r+r_{1} d_{1}$, while chains in $\mathcal{L}(M)$ have length at most $r+r_{1} d_{1}$. If $\operatorname{Leib}\left(L^{\prime}\right)=M^{\prime}$, then $M$ also has the $\left(r+r_{1} d_{1}\right)$-dimensional vector space lattice. But $M$ has at most two maximal subalgebras, one containing $B$ and $M \cap V$. This requires $\operatorname{dim}(M)=1$ and $\operatorname{dim}(L)=2$. In this case, $L \simeq L^{\prime}$. If $\operatorname{dim}(L)>2$, then $\operatorname{Leib}\left(L^{\prime}\right)=V^{\prime}$, and the signature of $L^{\prime}$ can be read from the length of the chain $L^{\prime} \div B^{\prime}$ and the dimensions of $B^{\prime} \cap V^{\prime}$ and $V^{\prime}$.

Lemma 2.7. Let $L$ be a one-generator Leibniz algebra and suppose that the number $k$ of chains is greater than 1 . Then $L^{\prime}$ is a one-generator algebra with the same signature as $L$ and $\phi(\operatorname{Leib}(L))=\operatorname{Leib}\left(L^{\prime}\right)$. 
Proof. Let $\left[r\left|r_{1}, \ldots, r_{k}\right| d_{1}, \ldots, d_{k}\right]$ be the signature of $L$. For each $i$, we have a single-chain subalgebra $C_{i} \supset B$ with signature $\left[r\left|r_{i}\right| d_{i}\right]$ such that $L \div B$ is the product of the chains $C_{i} \div B$.

I prove first that $B^{\prime} \nsubseteq \operatorname{Leib}\left(L^{\prime}\right)$. For this, it is sufficient to consider the case $k=2$. If $r>0$ or for any $i$, we have $r_{i} d_{i}>1$, then by Lemma 2.6, $B^{\prime} \nsubseteq \mathbb{L e i b}\left(L^{\prime}\right)$. So suppose that $r=0$ and that $r_{i}=d_{i}=1$ for $i=1,2$. Then $\mathcal{L}(L)$ is

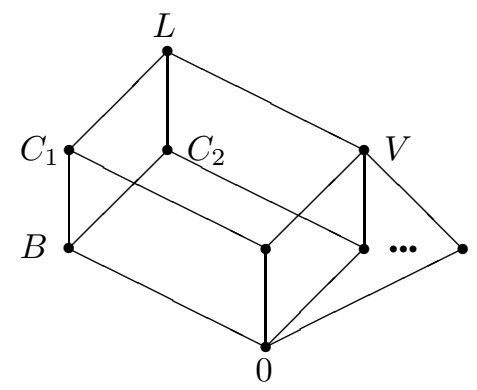

As $C_{1}, C_{2}$ do not have vector space lattices, Leib $\left(L^{\prime}\right)$ cannot be $C_{1}^{\prime}$ or $C_{2}^{\prime}$. If $B^{\prime} \subseteq \operatorname{Leib}\left(L^{\prime}\right)$, then $B^{\prime}=\operatorname{Leib}\left(L^{\prime}\right)$ and $L^{\prime} / B$. is a Lie algebra, contrary to $L^{\prime} \div B^{\prime}$ being the diamond lattice.

We now have, whatever the signature of $L$, that $\phi(\operatorname{Leib}(B)), \phi\left(\operatorname{Leib}\left(C_{i}\right)\right)$ are all contained in $\operatorname{Leib}\left(L^{\prime}\right)$. But they generate $V^{\prime}$, so $V^{\prime} \subseteq \operatorname{Leib}(L)$. As $V^{\prime}$ is a maximal subalgebra of $L^{\prime}$, this implies that $V^{\prime}=\operatorname{Leib}\left(L^{\prime}\right)$.

Take an element $b^{\prime}$ which generates $B^{\prime}$ and let $\beta: V^{\prime} \rightarrow V^{\prime}$ be left multiplication by $b^{\prime}$. Then the minimum polynomial of $\beta \mid C_{i}^{\prime} \cap V^{\prime}$ is $x^{r} q_{i}^{r_{i}}(x)$ for some irreducible polynomial $q_{i}(x)$. I now prove that the $q_{i}$ are distinct.

Suppose that $q_{1}=q_{2}$. Let $W_{i}$ be a minimal invariant subspace of $C_{i}^{\prime} \cap V^{\prime}$ with minimum polynomial $q_{i}(x)$. Then $W_{1} \simeq W_{2}$ as $F[\beta]$-modules. Take an isomorphism $\gamma: W_{1} \rightarrow W_{2}$ and put $W^{*}=\left\{w+\gamma(w) \mid w \in W_{1}\right\}$. Then $W^{*}$ is an invariant subspace and $B^{\prime}+W^{*}$ is a subalgebra not in the product of chains. Therefore $q_{1} \neq q_{2}$.

We now have that $\beta^{r} V^{\prime}$ is generated as $F[\beta]$-module by some single element $w$, and it follows that $\operatorname{alg}\left\langle b^{\prime}+w\right\rangle=L^{\prime}$.

\section{Recognising Leib $(L)$}

In this section, $L, L^{\prime}$ are Leibniz algebras and $\phi: \mathcal{L}(L) \rightarrow \mathcal{L}\left(L^{\prime}\right)$ is a lattice isomorphism. The aim is to prove that $\phi(\operatorname{Leib}(L))=\operatorname{Leib}\left(L^{\prime}\right)$. For this to fail, we must have a diamond subalgebra $\langle b, v\rangle, b v=v$, with $\langle b\rangle^{\prime} \subseteq \operatorname{Leib}\left(L^{\prime}\right)$. I shall assume this and show that, if $\operatorname{dim}(L) \geq 3$, then there are other subalgebras whose relation to $\langle b, v\rangle$ makes this impossible. It is convenient to represent data as a geometric configuration, with points representing 1-dimensional subalgebras and lines representing 2-dimensional subalgebras, all of whose subspaces are subalgebras. Thus the lines represent Lie subalgebras. Broken lines are used to represent 2-dimensional subalgebras which have subspaces that are not subalgebras. Their lattice of subalgebras is the diamond lattice, the product of two chains each of length 1 .

Theorem 3.1. Let $L, L^{\prime}$ be Leibniz algebras and let $\phi: \mathcal{L}(L) \rightarrow \mathcal{L}\left(L^{\prime}\right)$ be a lattice isomorphism. Suppose $\operatorname{dim}(L) \geq 3$. Then $\left.\phi(\operatorname{Leib}(L))=\operatorname{Leib}\left(L^{\prime}\right)\right)$. 
Proof. In the notation set out above, I assume that $\langle b\rangle^{\prime} \subseteq \operatorname{Leib}\left(L^{\prime}\right)$. I investigate and eliminate a number of cases.

Case 1: Suppose that there exists $x \in L, x^{2}=x b=b x=x v=v x=0$. Since $(b+\lambda x) v=v$, we have that $\langle(b+\lambda x), v\rangle$ is a diamond subalgebra for all $\lambda \in F$.

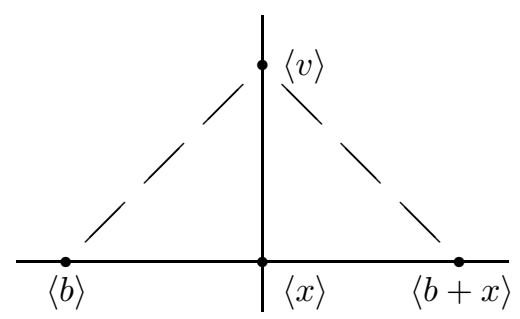

For suitable choice of $b^{\prime}$ and $x^{\prime}$, we have $b^{\prime}+x^{\prime} \in\langle b+x\rangle^{\prime}$. Since $b^{\prime} \in \operatorname{Leib}(L)$, we can choose $v^{\prime}$ such that $v^{\prime} b^{\prime}=b^{\prime}$. Since $\left\langle v^{\prime}, b^{\prime}+x^{\prime}\right\rangle$ is a diamond algebra, we must have $v^{\prime}\left(b^{\prime}+x^{\prime}\right)=\lambda\left(b^{\prime}+x^{\prime}\right)$ for some $\lambda \in F$. But $b^{\prime},\left(b^{\prime}+x^{\prime}\right) \in \operatorname{Leib}\left(L^{\prime}\right)$, so $x^{\prime} \in \operatorname{Leib}\left(L^{\prime}\right)$. Since $\left\langle v^{\prime}, x^{\prime}\right\rangle$ is a Lie algebra, $v^{\prime} x^{\prime}=-x^{\prime} v^{\prime}=0$ and $v^{\prime}\left(b^{\prime}+x^{\prime}\right)=b^{\prime}$ contrary to $v^{\prime}\left(b^{\prime}+x^{\prime}\right)=\lambda\left(b^{\prime}+x^{\prime}\right)$. Thus Case 1 is impossible.

Case 2: Suppose that $\operatorname{dim}(\operatorname{Leib}(L))>1$. Then there exists $w \in \operatorname{Leib}(L)$ not in $\langle v\rangle$. If in the space $W$ generated by $w$ under the action $\theta$ of $b$ contains an element $w_{0}$ such that $\theta w_{0}=0$, then we have Case 1 . Therefore $\theta$ acts non-singularly on $W$ and $\operatorname{alg}\langle b+w\rangle \supset W$. If $\operatorname{dim}(W)>1$, then we cannot have $b^{\prime} \in \operatorname{Leib}\left(L^{\prime}\right)$, so $b w=\lambda w$, $\lambda \neq 0$. If $\lambda \neq 1$, then for every $\mu \neq 0, b$ and $v+\mu w$ generate a 3-dimensional subalgebra. If $\lambda=1$, then for all $\mu, b$ and $v+\mu w$ generate a diamond algebra.

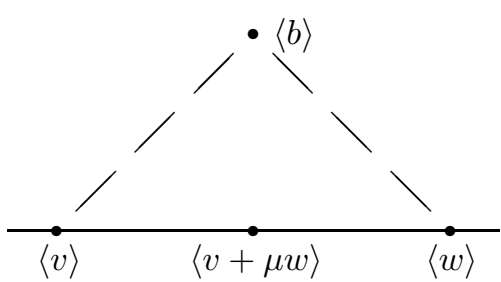

Case $2(\mathrm{a}): \lambda \neq 1$

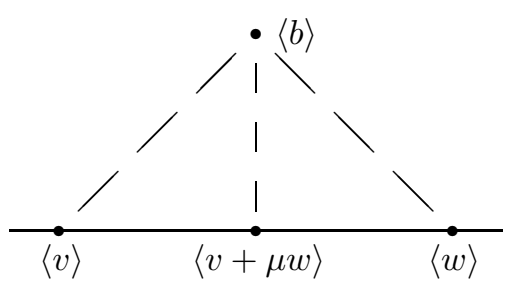

Case 2(b): $\lambda=1$

Since $b^{\prime} \in \operatorname{Leib}\left(L^{\prime}\right)$, for suitable choice of $v^{\prime}, w^{\prime}$, we have $v^{\prime} b^{\prime}=b^{\prime}$ and $w^{\prime} b^{\prime}=b^{\prime}$. But this implies that $\left(v^{\prime}-w^{\prime}\right)$ and $b^{\prime}$ generate a 2-dimensional Lie algebra, contrary to the lattice information. Therefore Case 2 is impossible.

Case 3: $\operatorname{dim}(\operatorname{Leib}(L))=1$. Take $x \notin\langle b, v\rangle$. If $x^{2} \neq 0$ but $x^{3}=0$, then $\phi\left(\left\langle x^{2}\right\rangle\right) \subseteq \operatorname{Leib}\left(L^{\prime}\right)$ contrary to assumption. Therefore either $x^{2}=0$ or $\langle x, v\rangle$ is the diamond algebra. In either case, there exists $c=x+\lambda v$ with $c^{2}=0$. Either $c v=v$ or $c v=0$. Since $v^{\prime} \notin \operatorname{Leib}\left(L^{\prime}\right)$, if $c v=v$, then $c^{\prime} \in \operatorname{Leib}\left(L^{\prime}\right)$ and $\operatorname{dim}\left(\operatorname{Leib}\left(L^{\prime}\right)\right)>1$, contrary to Case 2 applied to $\phi^{-1}$. So $c v=0$. But this implies that $x^{2}=x v=0$. Therefore, for every $x \notin\langle b, v\rangle$, we have $x v=0$. But $x v=0$ and $(x+b) v=0$ implies $b v=0$ contrary to assumption. Thus Case 3 also is impossible. 


\section{REFERENCES}

1. D. W. Barnes, Lattice isomorphisms of Lie algebras, J. Austral. Math. Soc. 4 (1964), 470-475.

2. D. W. Barnes, Lattice automorphisms of semi-simple Lie algebras, J. Austral. Math. Soc. 16 (1973), 43-53.

3. D. W. Barnes, Schunck classes of soluble Leibniz algebras, arXiv:1101.3046 (2011)

4. M. Goto, Lattices of subalgebras of real Lie algebras, J. Algebra 11 (1969) 6-24.

5. D. A. Towers, Dimension preserving lattice isomorphisms of Lie algebras, Comm. Algebra 8 (1980), 1371-1386.

6. D. A. Towers, Lattice automorphisms of Lie algebras, Arch. Math. (Basel) 46 (1986), 39-43.

7. D. A. Towers, On semi-modular subalgebras of Lie algebras over fields of arbitrary characteristic Asian-Eur. J. Math. 1 (2008), 283-294.

1 Little Wonga Rd, Cremorne NSW 2090 Australia

E-mail address: donwb@iprimus.com.au 